\title{
HJ16 induced resistant virus displays rare natural mutation highlighting the conserved nature of this new CD4bs epitope
}

\author{
Sunita S Balla-Jhagihoorsingh ${ }^{1 *}$, Betty Willems ${ }^{1}$, Katleen Vereecken ${ }^{1}$, Davide Corti ${ }^{2}$, Leo Heyndríckx', \\ Guido Vanham", The BMGF CAVD UCL Vaccine Discovery Consortium
}

From Frontiers of Retrovirology 2011

Amsterdam, The Netherlands. 3-5 October 2011

\section{Background}

Immunogen development for HIV-1 vaccines can be based on epitope identification of naturally occurring neutralizing antibodies in HIV-1 infected patients. A neutralizing monoclonal antibody, $\mathrm{HJ} 16$, was obtained at IRB from a patient recruited at ITM which recognized a new epitope in the CD4bs and neutralized mostly tier 2 strains that were not neutralized by b12[1]. This new CD4bs epitope is not fully characterized yet but could be important for immunogen design. We therefore used a neutralization sensitive virus and after resistance induction compared the resistant and sensitive strains to map important regions for the HJ16 neutralizing activity.

\section{Materials and methods}

HJ16 resistance was induced by culturing the sensitive replication competent VI1090 (CRFO2_AG) strain (IC50 $0.10 \mu \mathrm{g} / \mathrm{ml}$ ) in increasing amounts of $\mathrm{HJ} 16$ on freshly isolated PBMC until a resistant strain was obtained that was able to replicate in almost $200 \mu \mathrm{g} / \mathrm{ml} \mathrm{HJ16}$. Neutralizing activity was measured using TZMbl and PBMC neutralization assays. Site-directed mutagenesis was carried out to induce the observed mutations into the VI1090 expressing vector.

\section{Results}

Sequencing of the HJ16 sensitive versus the resistant strain revealed a distinct point mutation where the neutral asparagine was replaced by the negative charged aspartic acid. This N276D point mutation is only seen in $0.69 \%$ of the group $M$ strains described in the Los Alamos database (total of 1885 viruses, 6 cases with N276D and 7 cases with a polar N276S mutation). Introduction of this mutation into the HJ16 neutralization sensitive construct rendered the mutated strain resistant to neutralization by at least 100 fold higher HJ16 concentration.

\section{Conclusions}

Results show that the mutation in the N-linked glycosylation site at $\mathrm{N} 276$ has a distinct influence on sensitivity to the HJ16 CD4bs antibody. Although it is disappointing that a single mutation seems to induce resistance it is obvious that this mutation is quite unique and occurs rarely in natural infection rendering this epitope very suitable for further vaccine development.

\section{Author details}

${ }^{1}$ Institute of Tropical Medicine (ITM), Antwerp, Belgium. ${ }^{2}$ Institute for Research in Biomedicine (IRB), Bellinzona, Switzerland.

Published: 3 October 2011

\section{Reference}

1. Corti D, Langediuijk JP, Hinz A, Seaman MS, Vanzetta F, FernandezRodriguez BM, Silacci C, Pinna D, Jarrossay D, Balla-Jhagjhoorsingh S, et al: Analysis of memory $B$ cell responses and isolation of novel monoclonal antibodies with neutralizing breadth from HIV-1 -infected individuals. PLoS One 2010, 5:e8805.

doi:10.1186/1742-4690-8-S2-P33

Cite this article as: Balla-Jhagihoorsingh et al:: HJ16 induced resistant virus displays rare natural mutation highlighting the conserved nature of this new CD4bs epitope. Retrovirology 2011 8(Suppl 2):P33.

${ }^{1}$ Institute of Tropical Medicine (ITM), Antwerp, Belgium

Full list of author information is available at the end of the article

(c) 2011 Balla-Jhagihoorsingh et al; licensee BioMed Central Ltd. This is an open access article distributed under the terms of the 